\title{
Minimizing non-radiative recombination losses in perovskite solar
}

\section{cells}

Deying Luo $^{1 \dagger}$, Rui Su${ }^{1 \dagger}$, Wei Zhang ${ }^{2,3^{*}}$, Qihuang Gong ${ }^{1,4}$, Rui Zhu ${ }^{1,4^{*}}$

${ }^{1}$ State Key Laboratory for Artificial Microstructure and Mesoscopic Physics, School of Physics, Frontiers Science Center for Nano-optoelectronics \& Collaborative Innovation Center of Quantum Matter, Peking University, Beijing 100871, China

${ }^{2}$ Advanced Technology Institute, University of Surrey, Guildford GU2 7XH, United Kingdom

${ }^{3}$ State Centre for International Cooperation on Designer Low-Carbon and Environmental Material (SCICDLCEM), School of Materials Science and Engineering, Zhengzhou University, Zhengzhou 450001, P. R. China

${ }^{4}$ Collaborative Innovation Center of Extreme Optics, Shanxi University, Taiyuan, Shanxi 030006, China

These authors contributed equally to this work: Deying Luo and Rui Su

*Corresponding author: E-mail: iamzhurui@pku.edu.cn (R.Z.); wz0003@surrey.ac.uk (W.Z.).

Abstract| Photovoltaic solar cells based on metal halide perovskites have gained considerable attention over the past decade because of their potentially low production cost, earth-abundant raw materials, ease of fabrication and ever-increasing power conversion efficiencies of up to $25.2 \%$. This type of solar cells offers the promise of generating electricity at a more competitive unit price than traditional fossil fuels by 2035 . Nevertheless, the best research cell efficiencies are still below the theoretical limit defined by the Shockley-Queissier theory owing to the presence of non-radiative recombination losses. In this Review, we analyse the predominant pathways that contribute to nonradiative recombination losses in perovskite solar cells, and evaluate their impact on device performance. We then discuss how non-radiative recombination losses can be estimated through reliable characterization techniques, and highlight some notable advances in mitigating these losses, which hint at pathways towards defect-free perovskite solar cells. 
Finally, we outline directions for future work that will push the efficiency of perovskite solar cells towards the radiative limit.

\section{[H1] Introduction}

Metal halide perovskites (BOX 1) are the focus of one of the most active areas of research in the effort to harness solar energy by highly efficient and inexpensive photovoltaic technologies ${ }^{1-4}$. A sharp increase in power conversion efficiencies (PCEs) from an initial $3.8 \%^{3}$ to the most recently certified value of $25.2 \%^{5}$ has been achieved within a decade using single-junction perovskite solar cells (PSCs). The best current PCE is comparable to those of some commercially available products on the photovoltaic market, such as crystalline silicon and copper indium gallium selenide (CIGS) solar cells. All-perovskite tandem PSCs have also yielded remarkable PCEs exceeding 25\% for fourterminal configurations ${ }^{6}$, whereas the efficiency of perovskite-on-silicon tandem solar cells has surpassed $28 \%{ }^{7}$. We attribute these notable progresses to the outstanding properties of metal halide perovskites, and to lessons quickly learned from other photovoltaic technologies, such as dye-sensitized solar cells, organic photovoltaics and silicon solar cells. Nevertheless, present record PCEs of PSCs are still behind the radiative limit defined by the Shockley-Queissier (SQ) theory. The radiative limit assumes that all recombination processes within a solar cell are radiative and that all emitted photons can escape from the cell. In this context, non-radiative recombination losses are negligible and do not limit device performance.

However, non-radiative recombination losses are inevitable in all types of solar cells owing to defect-assisted recombination, Auger recombination, electron-phonon 
coupling and band-tail recombination ${ }^{8}$. These losses cause the record device performance parameters, which include open-circuit voltage $\left(V_{\mathrm{oc}}\right)$, short-circuit current density $\left(J_{\mathrm{sc}}\right)$, fill factor and PCE, to deviate from the radiative limit. Moreover, the presence of a considerable density of non-radiative recombination centers normally accelerates the degradation process of perovskite absorbers ${ }^{9,10}$, hindering their development for practical applications. To further boost device performances towards the SQ radiative limit and simultaneously enhance the long-term stability of solar cells, it is essential to minimize all types of non-radiative recombination losses.

Under continuous sunlight illumination, electrons are excited from the valence band of a semiconductor absorber to its conduction band, splitting the quasi-Fermi levels of electrons $\left(E_{\mathrm{Fn}}\right)$ and holes $\left(E_{\mathrm{Fp}}\right)$. The difference between quasi-Fermi levels for electrons and holes sets the achievable value of $V_{\mathrm{oc}}$ for a solar cell ${ }^{11,12}$. Any non-radiative recombination loss reduces the splitting of quasi-Fermi levels, lowering the achievable $V_{\mathrm{oc}}$. The $V_{\text {oc }}$ deficit, defined as $E_{\mathrm{g}} / q-V_{\mathrm{oc}}\left(E_{\mathrm{g}}\right.$ is the optical bandgap of the perovskite material, $q$ the elementary charge) is normally used to quantify the decrease in $V_{\text {oc. }}$ Recently, tremendous efforts have been devoted to reducing the $V_{\text {oc }}$ deficit and improving PCEs, including (but not limited to) crystallization control $^{13,14}$, defect passivation ${ }^{15,16}$, interface engineering $^{15,17-20}$ and formation of graded junctions ${ }^{21,22}$. Yet, the $V_{\text {oc }}$ deficit of best performing PSCs, at $0.34-0.41 \mathrm{~V}$, is still above the losses taken into account in the SQ theory $(\sim 0.27 \mathrm{~V})^{14,20,23,24}$.

In this Review, we summarize the current understanding of the origin and nature of non-radiative recombination losses in PSCs, with a particular focus on intrinsic defectassisted recombination, interface-induced recombination, electron-phonon coupling and 
band-tail recombination. These types of losses have a substantial impact on the electronic and optoelectronic properties of both perovskite bulk materials and material interfaces in PSCs. We also discuss some measurement techniques that can be utilized to quantify nonradiative recombination losses, and highlight recent advances in the reduction of such losses in PSCs to enhance device performance and long-term stability. We finally outline the steps that should be undertaken to minimize non-radiative recombination losses and boost PCEs to reach the theoretical radiative limit.

\section{[H1] Device configuration and efficiency}

\section{[H2] Device configurations of PSCs}

An efficient PSC comprises an intrinsic perovskite absorber (i) sandwiched between a positive (p) and a negative (n) charge extraction layer ${ }^{25-26}$, which in this Review we call p-type and n-type layers, respectively. PSCs are generally classified into three categories: mesoporous n-i-p, planar n-i-p and planar p-i-n (also called inverted planar) PCSs (FIG. 1). To date, n-i-p PSCs (both mesoporous and planar) exhibit superior PCEs as compared to $\mathrm{p}-\mathrm{i}-\mathrm{n}$ PSCs ${ }^{19,27}$. Nevertheless, $\mathrm{p}-\mathrm{i}-\mathrm{n}$ PSCs have attracted attention because of their ease of fabrication, compatibility with flexible substrates, versatile energy-band engineering and the possibility of fabricating tandem cells ${ }^{21,28,29}$.

\section{[H2] Theoretical efficiencies}

When a single-junction solar cell absorbs radiation from the sun (air mass (AM) $1.5 \mathrm{G}$ ) and its environment (temperature $T=300 \mathrm{~K}$ ), the maximum achievable PCE and the $V_{\mathrm{oc}}$ at the radiative limit ( $\left.V_{\mathrm{oc}, \mathrm{rad}}\right)$ as a function of bandgap can be obtained by using the SQ theory, also known as the detailed balance theory ${ }^{30,31}$ (see Supplementary Information for 
the achievable PCEs and $V_{\mathrm{oc}}$, rad). Calculated PCEs as a function of bandgap and record efficiencies achieved for a variety of solar cells are shown in FIG. 1b. The bandgaps of metal halide perovskites (typically $E_{\mathrm{g}} \sim 1.60 \mathrm{eV}$ ) can be engineered by utilizing mixed ions at the $\mathrm{A}, \mathrm{B}$, and $\mathrm{X}$ sites. For example, mixed-cation halide perovskites (such as $\mathrm{FA}_{0.92} \mathrm{MA}_{0.08} \mathrm{PbI}_{3}, E_{\mathrm{g}} \sim 1.50 \mathrm{eV}$ ) have been realized by mixing FA and MA cations at the A site, delivering a PCE exceeding $23 \%{ }^{4}$. However, this value is still far from the theoretical efficiency limit of $\sim 32 \%$ for a 1.50 -eV bandgap perovskite (FIG. 1b). State-of-the-art PSCs have much lower $V_{\text {oc }}$ deficits than organic and dye-sensitized solar cells, but higher than GaAs and GAInP solar cells (FIG. 1c), which are close to the $V_{\mathrm{oc}}$, rad limit. PSCs haven't yet reached the $V_{\mathrm{oc}}$, rad limit primarily owing to recombination losses of carriers in the complete cell. We believe that exploring the origins of recombination losses will help to narrow this gap and improve device performances.

\section{[H1] Non-radiative recombination pathways}

\section{[H2] Carrier generation and recombination}

An ideal photovoltaic material has a high absorption coefficient to efficiently harvest incident photons and convert them into free charge carriers. The absorption coefficients of perovskite absorbers are roughly $10^{5} \mathrm{~cm}^{-1}$ owing to the direct transition between energy bands ${ }^{32}$. Though recent works have revealed the presence of bandgaps with direct-indirect character ${ }^{33}$ and a shift of minimum energy points in reciprocal space induced by the Rashba effect (spin-orbit coupling) ${ }^{34}$, their impact on the light absorption process is negligible. In reality, the photon harvesting process in a complete PSC is unavoidably accompanied by optical losses ${ }^{35}$, which include reflection losses and parasitic absorption at the semi-transparent electrodes and charge extraction layers. Strategies to 
minimize the optical losses include the use of high-transmittance semi-transparent electrodes and charge-extraction layers, and the addition of an anti-reflection layer comprised of magnesium fluoride ${ }^{36,37}$. The remaining challenge is to lower the reflection losses at the bottom interface on which the perovskite film is deposited, which arise from the mismatch between the optical refractive index of the perovskite and bottom extraction layer.

Following light absorption, hot carriers (carriers with energies greater than the bandgap) thermalize — that is, they exchange energy with one another - and $\operatorname{cool}^{38-41}$. The ground and excited states exist in a superposition for a given dephasing time (for example $220 \mathrm{fs}$ for $\left.\mathrm{MAPbI}_{3}\right)^{42}$. After thermalization (10-85 fs), hot carriers start cooling down to the conduction band minimum and release thermal energy ${ }^{43}$ (FIG. 2a). The hotcarrier lifetime of a perovskite absorber is expected to be on the 100 -fs timescale ${ }^{44}$. The prolonged hot-carrier cooling time is correlated with carrier concentration ${ }^{44}$, phonon bottleneck effect ${ }^{39}$, large polaron character ${ }^{45}$ and electrostatic screening ${ }^{46}$.

After the hot-carrier cooling, carrier recombination processes, which include radiative and non-radiative recombination, compete with each other. The whole recombination process can be described $\mathrm{as}^{47}$ :

$$
\frac{d n}{d t}=-\kappa_{1} n-\kappa_{2} n^{2}-\kappa_{3} n^{3}
$$

Where $\kappa_{1}, \kappa_{2}$ and $\kappa_{3}$ are the first-, second- and third-order rate constants associated with defect-assisted (monomolecular), radiative (bimolecular), and Auger (three body) recombination processes, respectively; $\mathrm{n}$ is the photo-generated carrier density. These recombination rate constants can be determined by photoluminescence decay and by pumpprobe spectroscopy ${ }^{48}$. Defect-assisted recombination and Auger recombination contribute 
non-radiative recombination losses. Defect-assisted recombination depends on the energy depths and the density of defects, whereas Auger recombination predominantly occurs for perovskite absorbers with high carrier concentrations $\left(>10^{17} \mathrm{~cm}^{-3}\right)^{48}$. The major nonradiative recombination pathways, including intrinsic defect-assisted recombination, Auger recombination, interface-induced recombination and band-tail recombination ${ }^{8}$, along with the radiative recombination process, are shown in FIG. $2 b-d$. Recent studies have confirmed that Auger recombination in PSCs may be secondary under AM 1.5G illumination (with carrier concentrations in the range of $\sim 10^{13}$ to $10^{15} \mathrm{~cm}^{-3}$ ) 49 . The origin of other non-radiative recombination losses in PSCs is discussed in detail below.

\section{[H2] Defect-assisted recombination losses}

In PSCs, photo-generated carriers must have sufficiently long lifetimes to be collected by the electrodes before non-radiative recombination occurs (see Supplementary Information for carrier lifetimes and their contributions to the $V_{\mathrm{oc}}$ ). Non-radiative recombination decays are accelerated by scatterers that include defects ${ }^{13}$, phonons $^{50}$, impurities $^{51}$ and mobile species ${ }^{52,53}$, lowering the $V_{\text {oc. }}$. Deep-level defects (that is, those with a thermal activation energy higher than $k_{\mathrm{B}} T$, where $k_{\mathrm{B}}$ is the Boltzmann constant) are the predominant trap sources for non-radiative recombination losses, whereas shallow-level defects have a negligible impact on non-radiative decays and $V_{\mathrm{oc}}{ }^{9}$.

In a perovskite thin film, the most widely studied defects are intrinsic point defects, which can have different origins ${ }^{54-56}$ and include interstitials (such as $\mathrm{MA}_{\mathrm{i}}, \mathrm{Pb}_{\mathrm{i}}$ and $\left.\mathrm{I}_{\mathrm{i}}\right)^{55}$, vacancies (such as $\mathrm{V}_{\mathrm{MA}}, \mathrm{V}_{\mathrm{Pb}}$ and $\mathrm{V}_{\mathrm{I}}{ }^{20}$ and antisites (such as MAPb, MA $\mathrm{A}_{\mathrm{I}}, \mathrm{Pb}_{\mathrm{MA}}, \mathrm{Pb}_{\mathrm{I}}, \mathrm{I}_{\mathrm{MA}}$ and $\mathrm{IPb}^{57,58}$. In perovskites, these point defects are almost-positive or almost-negative 
charged defects, that is, they have holes or electrons localized in their vicinity. Firstprinciple calculations predict that most point defects (such as $\mathrm{MA}_{\mathrm{i}}, \mathrm{I}_{\mathrm{i}}, \mathrm{V}_{\mathrm{Pb}}, \mathrm{V}_{\mathrm{MA}}, \mathrm{V}_{\mathrm{I}}, \mathrm{MA} \mathrm{Pb}$ and $\mathrm{MA}_{\mathrm{I}}$ ) have low formation energies and can form shallow states in the bandgap. By contrast, deep-level defects (such as $\mathrm{Pb}_{\mathrm{i}}, \mathrm{Pb}_{\mathrm{MA}}, \mathrm{Pb}_{\mathrm{I}}, \mathrm{I}_{\mathrm{MA}}$ and $\mathrm{I}_{\mathrm{Pb}}$ ) have higher formation energies at room temperature ${ }^{47}$. Nonetheless, in some growth conditions the formation energies of $\mathrm{Pb}_{\mathrm{I}}$ and $\mathrm{I}_{\mathrm{MA}}$ might be low enough for deep-level recombination centers to form. Experimental results have further confirmed that deep-level defects exist in perovskite films ${ }^{15,60}$, and halide vacancies ( such as $\mathrm{V}_{\mathrm{I}}$ ) are the predominant contributors ${ }^{5,8}$. We attribute the discrepancy between theoretical calculations and experimental observations regarding halide vacancies to oversimplified defect calculations and diverse experimental conditions. The variety of perovskite chemical compositions and fabrication methods leads to complex defect formation processes. The next key step is to unambiguously identify the contribution of each type of defect to non-radiative recombination losses in a complete PSC, in particular for defects at the surface regions ${ }^{61,62}$.

\section{[H2] Interface-induced recombination losses}

Interface-induced recombination caused by mismatched energy-level alignment, surface defects and charge-carrier back transfer is another important source of non-radiative recombination $\operatorname{losses}^{63-67}$ (FIG. 2c). A perfect interface must selectively extract the majority carriers while blocking the minority carriers by means of a large Schottky barrier at the heterojunction ${ }^{68-70}$, with minimal defects and surface states at the interface ${ }^{71}$. In complete PSCs, if the energy barrier for electrons at the perovskite/electron-extraction layer heterojunction is over $200 \mathrm{meV}$, the electron exaction is blocked ${ }^{72}$. In addition, a recent study has shown that any finite energy barrier for holes at the perovskite/hole- 
extraction layer heterojunction can trigger band bending near the contacts, leading to an exponential increase in the recombination rates ${ }^{72}$. Both factors significantly contribute to the interfacial recombination currents in complete PSCs, leading to $V_{\text {oc }}$ values that deviate from the difference between $E_{\mathrm{Fn}}$ and $E_{\mathrm{Fp}}$. Moreover, undesirable surface defects and surface states further increase non-radiative recombination at the interfaces ${ }^{73,74}$.

An efficient charge-extraction layer must be able to maintain the difference between the quasi-Fermi levels while ensuring sufficient majority carrier extraction. The key step is to develop charge-extraction layers with the appropriate energy levels and a low density of defects. For the bottom interface close to the window electrode (fluorine-doped tin oxide or indium tin oxide, ITO, glass), for example, low-temperature solution-processed $\mathrm{SnO}_{2}$ has become increasingly popular as the electron extraction layer for n-i-p PSCs ${ }^{53,75,76}$. Compared with the traditional electron extraction layer $\mathrm{TiO}_{2}, \mathrm{SnO}_{2}$ possesses a favourable band alignment with the perovskite absorbers, yielding exceptionally high $V_{\mathrm{oc}}$ and PCEs ${ }^{19}$. For planar p-i-n PSCs, the organic polymeric semiconductor poly[bis (4-phenyl)(2,4,6trimethylphenyl)amine] (PTAA) has proven to be an excellent hole extraction layer while blocking electron injection from the perovskite. Recent research on recombination losses at the bottom interface has shown that most commonly used bottom charge extraction layers do not introduce additional losses compared with the isolated perovskite films ${ }^{72}$, whereas losses from the top charge extraction layers are a limiting factor for the $V_{\mathrm{oc}}$. Strategies to improve the interfaces are discussed in the section on defect passivation and interface engineering.

\section{[H2] Other recombination losses}

In addition to the recombination losses in the perovskite layer and at the interfaces, 
there are other recombination loss pathways to consider. Although the large polarons in perovskites can screen Coulomb potentials and even avoid scattering with defects and other impurities $^{77,78}$, free carrier transport processes are inevitably influenced by the electronphonon coupling ${ }^{79-81}$, which limits charge carrier motilities. In-depth studies have suggested that scattering from longitudinal optical phonons via the Fröhlich interaction dominates the electron-phonon coupling in perovskites ${ }^{50}$, which can broaden the photoluminescence emission spectra and reduce the radiative efficiency. Typically, reducing the electron-phonon coupling by dielectric confinement results in enhanced photoluminescence intensity and sharper photoluminescence spectra ${ }^{82}$.

The SQ theory requires a perfect abrupt absorption to reach the radiative limit. In a realistic PSC, the perovskite absorber displays significant band-tail absorption or recombination near the band edge ${ }^{83}$, which contributes a path for band-tail losses (FIG. 2d). The steepness of the absorption spectrum below the bandgap can be characterized by the Urbach energy $\left(E_{\mathrm{u}}\right)$, which can be determined by photothermal deflection spectroscopy ${ }^{84}$ or external quantum efficiency (EQE) measurments ${ }^{85}$. Perovskite absorbers (with bandgap of $\sim 1.60 \mathrm{eV})$ have an $E_{\mathrm{u}}$ as low as $13 \mathrm{meV}$, comparable to that of other semiconductors $(7.5$ $\mathrm{meV}$ for $\mathrm{GaAs}$ and $9.6 \mathrm{meV}$ for $\mathrm{c}-\mathrm{Si})^{32}$. To quantify the contribution of the band-tail recombination to the $V_{\text {oc }}$ deficit, the $V_{\text {oc, rad }}$ can be estimated as ${ }^{86}$ :

$$
V_{o c, r a d}=\frac{k_{B} T}{q} \ln \left(\frac{\int E Q E(E) \times \phi_{A M 1.5}(E) d E}{\int E Q E(E) \times \phi_{B B}(E) d E}+1\right)
$$

where $\phi_{\mathrm{AM} 1.5}(E)$ and $\phi_{\mathrm{BB}}(E)$ are the solar $\mathrm{AM} 1.5 \mathrm{G}$ spectrum and Plank's black body radiation spectrum at $300 \mathrm{~K}$, respectively. Assuming all the absorbed photons can be converted into electricity, we can estimate the achievable $V_{\mathrm{oc}}$, rad from the absorption spectra via photothermal deflection spectroscopy measurements. In other words, we can obtain the 
dependence of $V_{\mathrm{oc}}$, rad on $E_{\mathrm{u}}$ using equation 2 (with a quantitative $E_{\mathrm{u}}$ ). A recent report has

confirmed that a decrease in $E_{\mathrm{u}}$ translates into an improvement in $V_{\mathrm{oc}}{ }^{7}$. The reduction in $V_{\text {oc, rad }}$ values is usually very small if $E_{\mathrm{u}}$ is smaller than $26.7 \mathrm{meV}$ (at $300 \mathrm{~K}$ ). For example, $V_{\text {oc, rad }}$ decreases by around $10 \mathrm{mV}$ for a $E_{\mathrm{u}}$ of $15 \mathrm{meV}$. However, $V_{\mathrm{oc}}$, rad decrease substantially when $E_{\mathrm{u}}$ is over $26.7 \mathrm{meV}^{7}$. Mitigating all the recombination pathways in the PSCs enables an increase in the difference between $E_{\mathrm{Fn}}$ and $E_{\mathrm{Fp}}$, and thus a higher $V_{\mathrm{oc}}$.

\section{[H1] Quantifying recombination losses}

It is a popular notion that a good solar cell is also a good light-emitting diode ${ }^{87}$. Thus, quantifying the $V_{\text {oc }}$ deficit has become a popular way to quantify the non-radiative recombination losses of a complete PSC. The $V_{\text {oc }}$ deficit can be determined by accurately measuring the material bandgap and the external quantum efficiency of emitted photons from an isolated film and/or a complete PSC. In this section, we present some quantitative techniques to measure defects and non-radiative losses, and we discuss some recent results.

\section{[H2] Determining the bandgap}

To quantify the $V_{\text {oc }}$ deficit, the first step is to accurately estimate the bandgap. Although the bandgap of a semiconductor is a very fundamental property, its accurate determination in perovskites, in particular in the presence of a considerable number of defects and variations in the film thickness, is challenging, resulting in under- and overestimations of the $V_{\mathrm{oc}}$ deficit in several publications. Several approaches are used to determine the bandgaps of perovskites, including Tauc plots, Kubelka-Munk methods and the onset or differential of EQE spectra. The Tauc plots and Kubelka-Munk methods make use of the absorption and reflection, respectively, of incident light in a broad spectral range 
to determine the bandgap. However, the presence of sub-band absorption in perovskites can lead to an underestimation of the bandgap for thicker films. Absorption from these electrically active sub-bands can also lead to a red shift of the onset of EQE spectra. The impact of sub-band absorption on the bandgap determination can be minimized by using the differential of EQE spectra ${ }^{86}$ :

$$
\begin{aligned}
& E_{g}=\int_{a}^{b} E_{g} P\left(E_{g}\right) d E_{g} / \int_{a}^{b} P\left(E_{g}\right) d E_{g} \\
& P\left(E_{g}\right)=\frac{d}{d E} E Q E(E)
\end{aligned}
$$

where $a$ and $b$ are set to the integration limits, $E$ is the photon energy, and $\mathrm{P}\left(E_{\mathrm{g}}\right)$ is the probability distribution function of the bandgap energies. To avoid additional noise, the photon energies for the calculation of $\mathrm{P}\left(E_{\mathrm{g}}\right)$ are taken equal to $50 \%$ of the $\mathrm{EQE}$ peak value. A bandgap determination procedure based on this approach is shown in Supplementary Fig. 1.

\section{[H2] Photoluminescence quantum efficiency}

The principle of optical reciprocity between absorption and emission introduced by Gustav Kirchhoff in the context of transformation of radiation energy was adapted to parameters relevant to solar cells by Uwe $\mathrm{Rau}^{88}$. The difference between quasi-Fermi levels for photo-generated electrons and holes, $\mu$, is coupled with the photoluminescence intensity by $^{89}$ :

$$
I_{P L}(E)=\frac{2 \pi E^{2} \alpha(E)}{h^{3} c^{2}} \frac{1}{\exp \left(\frac{E-\mu}{k_{B} T}-1\right)}
$$

where $c$ is the speed of light, $\alpha(E)$ the absorptivity, which depends on the photon's energy, and $h$ the Planck's constant. When all non-radiative recombination pathways are eliminated, charges present in semiconductors only recombine through the radiation of photons. 
The photoluminescence quantum yield (PLQY) is a key parameter to quantify nonradiative recombination losses in semiconductors. In a PLQY measurement (Fig. 3a), all possible states accessible by the incident photons are excited. Thus, the carrier transport imbalance issue can be neglected. Under an excitation intensity equivalent to 1 sun, a quantitative relationship between the PLQY and the achievable $V_{\mathrm{oc}}$ in perovskites is described by ${ }^{90}$ :

$$
V_{o c}=V_{o c, r a d}+\frac{k_{B} T}{q} \ln (P L Q Y)
$$

Thus, an increase in external PLQY at solar fluence (excitation density $10^{15} \mathrm{~cm}^{-3}$ ) from $1 \%$ to close to unity would lead to a $V_{\text {oc }}$ rise of $0.12 \mathrm{~V}$.

In general, the PLQY depends on a few factors including the quality of the perovskite film, the energy level of the charge extraction layer and additional recombination pathways at the perovskite/charge extraction layer interface. Subsequent escapes of photons out of the sample and parasitic absorption also contribute to the external PLQY ${ }^{90}$. The PLQY of samples comprising layered structures can be used to quantify the recombination losses in each individual layer. For instance, the recombination losses in a perovskite film can be quantified by measuring the PLQY of the isolated perovskite film deposited on a quartz substrate; when the charge extraction layers are in contact with the film, the reduction in the PLQY value reflects the recombination losses at the interfaces. Specifically, some defects can be introduced at the perovskite/charge extraction layer interface, and a large energy barrier (200 meV for electrons) can contribute to a substantial non-radiative recombination current ${ }^{72}$. To assess the recombination losses of a PSC using the PLQY, a complete or close-to-complete cell (for example ITO/PTAA/perovskite/PC ${ }_{61} \mathrm{BM}([6,6]$-phenyl-C61-butyric acid methyl ester)) should be 
used in the measurement ${ }^{29}$.

\section{[H2] External radiative efficiency}

The external radiative efficiency (ERE) of a complete PSC operating in forwardbias mode is extensively utilized to estimate the $V_{\text {oc }}$ deficit (Fig. 3b). The ERE is usually measured when the injection current (dark recombination current) equals the short-circuit current density under a 1-sun illumination. A high ERE value reflects a high fraction of the injection current resulting in photon emission ${ }^{91}$, suggesting low non-radiative recombination losses. In general, the ERE value of a complete PSC is lower than the external PLQY of the perovskite film in a close-to-complete device. This could be due to variations in the quasi-Fermi levels and their spatial distribution in the two cases. If quasiFermi levels are flat throughout a complete PSC, the ERE of a complete PSC under a forward bias should be comparable to the PLQY. Otherwise, the PLQY from a close-tocomplete cell can be larger than the ERE value. Moreover, the higher reabsorption and lower light out-coupling efficiency (20\%-25\% for a planar structure) of complete PSCs compared to close-to-complete devices can also contribute to ERE values lower than PLQY values $^{90,92}$. The $V_{\text {oc }}$ is predicted to be $\mathrm{e}^{14,93}$ :

$$
V_{o c}=V_{o c, r a d}+60 m V \log (E R E)_{T=300 K}
$$

The radiative limit is realized when the ERE is equal to one. A $V_{\mathrm{oc}}$ loses approximately 60 $\mathrm{mV}$ each time the ERE is reduced by an order of magnitude. The ERE of the current best PSC is $8 \%^{19}$, which is greater than that of $\mathrm{Si}$ solar cells with a record PCE of $26.7 \%^{94,95}$ $(\mathrm{ERE}=\sim 2 \%)$. The high ERE of $32.3 \%$ for GaAs solar cells (PCE 28\%) suggests that there is ample room to increase the PCEs of PSCs by improving the ERE ${ }^{95}$.

Two pitfalls of electroluminescence measurements in a complete PSC should be 
considered. Firstly, an imbalanced transport of the injected electrons and holes in a complete PSC usually occurs under a forward bias. Injected carriers prefer to occupy the lowest-energy states, hindering carrier transport in perovskite absorbers ${ }^{96}$. Secondly, ion migration induced by a forward electric field can produce internal electric fields ${ }^{97,98}$, which screen the external electric field responsible for electron and hole movement towards the emissive regions, increasing the uncertainty in the measurement of non-radiative recombination losses. A feasible strategy for the precise assessment of non-radiative recombination losses in a complete PSC is to compare the ERE with the measured PLQY.

\section{[H2] Thermal admittance spectroscopy}

Defect-assisted non-radiative recombination losses are of fundamental importance for the $V_{\mathrm{oc}}$ of PSCs under open-circuit conditions. The prerequisite for minimizing defectassisted recombination losses is to explore the types of defects and their energy depths. Thermal admittance spectroscopy uses electrical measurements to record the capacitance and conductance of a complete PSC as a function of frequency and temperature. This type of measurement provides steady-state information about the trap density of states (tDOS) within a complete PSC. For example, a deep defect cannot respond and contribute to the capacitance in a high-frequency region, in which the test frequency is faster than the emission rate $\mathrm{e}^{99,100}$. Increasing the temperature can vary the emission rate and modify the capacitance and conductance. Defects within perovskite films often produce multiple subband levels in the forbidden band, and perovskite films processed using different preparation processes normally exhibit varied defect populations. Inconsistent responses of the characteristic peak in the differential capacitance spectra as a function of frequency and temperature are interpreted as originating from various defect levels and 
populations ${ }^{60,101}$. The tDOS within the forbidden band that contributes to non-radiative recombination losses was found to be in the range of $10^{16}-10^{17} \mathrm{~cm}^{-3}$ using optical techniques $^{102}$. From thermal admittance spectroscopy measurements, it was found that there is a higher density of defect states (on the order of $\sim 10^{18} \mathrm{~cm}^{-3}$ ) in a complete PSC than the isolated perovskite film ${ }^{15}$. The tDOS originating from defects with various energy depths can be generally classified into three regions: band $1(0.35-0.40 \mathrm{eV})$, band $2(0.40-$ $0.50 \mathrm{eV})$ and band $3(>0.50 \mathrm{eV}$, Fig. 3c). These defect energy depths are very similar to those obtained in a deep-level transient spectroscopy study ${ }^{103}$. Some effective strategies, such as coating a $\mathrm{PC}_{61} \mathrm{BM}$ layer on top of the perovskite film, can reduce the tDOS of band 2 and band 3 by nearly two orders of magnitude, whereas band 1, which is close to the bandgap edge, is almost unaffected. Thermal admittance spectroscopy results show a substantial decrease in deep-level defect populations with surface passivation ${ }^{15,20}$, indicating that defects located at the surface and grain boundaries can result in deep-level traps. However, it is challenging to identify all defect types and their origin simply by thermal admittance spectroscopy. Scanning tunneling microscopy, which is a surfacesensitive technique, can provide accurate information about defects in perovskite single crystals $^{104,105}$, but cannot be used on high-roughness polycrystalline films.

\section{[H2] Advanced photoluminescence imaging}

The defect-rich, close-to-surface regions of the perovskite films should in principle accelerate non-radiative recombination in complete PSCs. The bulk defects are another predominant source of non-radiative recombination losses. All non-radiative recombination centers in the perovskite film are randomly distributed. To quantify the variations in their spatial distribution and to identify their origin in complete PSCs, a 
hyperspectral absolute photoluminescence imaging technique has been developed ${ }^{106}$. Photoluminescence imaging has originally been developed to evaluate the quality of thermally evaporated films ${ }^{107}$. Recently, this technique was used to quantify the nonradiative losses caused by bulk defects, buried bottom interfaces and top interfaces ${ }^{106}$ (FiG. 3d), and showed that the non-radiative recombination losses in the active area are remarkably non-uniform. Non-radiative recombination losses in the bulk material result in a decrease of the quasi-Fermi level splitting $(\sim 135 \mathrm{meV})$. After incorporating the charge extraction layers (PTAA as the hole extraction layer and $\mathrm{C}_{60}$ as the electron extraction layer), the interface-induced recombination leads to an $80-\mathrm{meV}$ decrease in the quasi-Fermi level splitting at each interface (FIG. 3e). This implies that the $V_{\text {oc }}$ deficits related to nonradiative recombination losses induced by each component do not add up in a complete PSC. The overall $V_{\text {oc }}$ deficit could be ascribed to an increase in the non-radiative recombination current, on which $V_{\mathrm{oc}}$ depends logarithmically. Hence, an in-depth understanding of the role of each individual recombination process within a complete PSC is essential for future work.

\section{[H1] Reducing non-radiative recombination losses}

We discussed the current understanding of the origin of all sorts of non-radiative recombination losses, along with their impact on device performance, the characterization techniques used to quantify them, and the possible pitfalls coming in the way of accurate measurements. In this section, we describe the approaches employed to mitigate nonradiative recombination losses, including the control of perovskite crystallization, defect passivation and interface engineering, and the formation of graded junctions. 


\section{[H2] Control of perovskite crystallization}

First and foremost, to minimize non-radiative recombination losses high-quality, ideally defect-free perovskite films are desirable. One effective means to achieve this goal is by controlling their crystallization. The parameters that influence crystallization include the chemical composition of the perovskite film, the solvent used to synthesize it, the substrate on which it is grown, the fabrication protocol used and environmental factors (such as atmosphere, pressure and gas flow rate).

To date, solvent engineering has been the most successful approach to obtain highquality films, enabling one-step deposition. A pioneering work used a mixed solvent of gamma-butyrolactone and dimethyl sulfoxide ${ }^{108}$, which resulted in noticeable improvements in the perovskite crystallization. Since then other solvents, including nmethyl-2-pyrrolidone ${ }^{109}$ and acetonitrile ${ }^{55}$, were mixed with the most widely used dimethylformamide in perovskite precursor solutions. Lessons learned from organic solar cells, such as using the additive 1,8-diiodooctane, proved useful to tune the crystallization of perovskites ${ }^{110}$. More recently, other additives have been reported to control perovskite crystallization, including 5-aminovaleric acid $^{111}$, methylammonium bromide ${ }^{112,113}$, methylammonium chlorine ${ }^{4,114}$ and $1,4,7,10,13,16$-hexaoxacyclooctadecane (crown) $)^{115}$.

Changes in device fabrication protocols have also improved perovskite crystallization $^{116,117,118,119}$. For example, a traditional fabrication route based on lead halide was replaced by a non-halide lead acetate route, which considerably improved the crystallization of perovskite films $^{29,120,121}$, reducing band-tail recombination losses. It has also been reported that the crystallization of perovskites correlates with the fabrication atmosphere (for example $\mathrm{N}_{2}, \mathrm{O}_{2}$, temperature and humidity) ${ }^{75,122}$. The presence of oxygen 
enables the formation of super-oxidized species at the surface through binding to iodide vacancies $^{122}$, enhancing the fraction of light emission from radiation processes. Recently, it turned out that forming perovskites in ambient air conditions (30-40\% humidity) can enhance the crystallization for two-step fabricated films ${ }^{19,75,117}$ and simultaneously reduce non-radiative recombination. Nevertheless, it is unclear if this observation can be extended to films processed using other protocols.

To further improve perovskite crystallization, the optimization of the perovskite composition by incorporating alkali halides, such as $\mathrm{CsI}$, RbI and $\mathrm{KI}$, has been explored $^{14,123}$. Alkali halides were found to function as mediators tuning the uniformity of halide anions over the substrate and mitigating the formation of defects ${ }^{124}$. The exact location of $\mathrm{Rb}^{+}$in perovskite absorbers is still debated ${ }^{125}$, but its incorporation has yielded a $V_{\mathrm{oc}}$ of $1.24 \mathrm{~V}$ for a perovskite with a bandgap of $1.63 \mathrm{eV}^{14}$. Moreover, $\mathrm{K}^{+}$probably accumulates at the surface and at grain boundaries, passivating defects at these sites (FIG. 4a), and $\mathrm{K}^{+}$-rich regions at these locations can regulate the dielectric confinement ${ }^{126}$, lowering non-radiative recombination losses. Such improvements resulted in a high external PLQY of $15 \%$ and a $V_{\text {oc }}$ deficit of $0.39 \mathrm{~V}$ (corresponding to a $1.56-\mathrm{eV}$ bandgap) $)^{13}$.

The long-term stability of PSCs under working conditions can also be remarkably improved by crystallization control, owing to the reduction of ion migration and halide segregation $^{13,124}$. Sodium fluoride has been proven to improve operational stability ${ }^{127}$, which has led to devices retaining $90 \%$ of their original PCEs after aging 1,000 hours at the maximum power point. The europium ion pair $\left(\mathrm{Eu}^{3+}-\mathrm{Eu}^{2+}\right)$ that serves as a redox shuttle selectively oxidizes neutral lead and reduces neutral iodide ${ }^{2}$. The resulting PSCs displayed substantially improved long-term stability under stressful tests of light soaking and heating 
at $85{ }^{\circ} \mathrm{C}$. The encapsulation of individual perovskite grains with amorphous silica layers has led to the suppression of perovskite decompositions and ion migration, contributing to a better operational stability ${ }^{128}$. Thus, developing new and effective approaches for crystallization control is expected to further reduce defects in PSCs and improve device performance.

\section{[H2] Defect passivation}

Defect-assisted recombination of photo-generated electrons and holes dominates the non-radiative recombination losses in a complete PSC. Defects in PSCs can be categorized as point defects (such as interstitials, antisites or vacancies), 2D extended defects (grains boundaries and surfaces) and 3D defects (such as iodine or lead clusters), plus additional defects at the contacts. Defects are highly dependent on the deposition process, and in most cases several types of defects can co-exist in a perovskite film. The selection of appropriate passivation methods is important to heal possible defects within the perovskite.

Organic molecules with specific functional groups (Fig. 4b) are used to passivate defects. Among them, choline halide was shown to passivate both positive and negative charged defects ${ }^{20}$, owing to the synergistic functions of the quaternary ammonium and halide ions. D-4-tert-butylphenylalanine (D4TBP), which contains both amine and carboxyl groups, was found to mitigate the point defects at grain boundaries and to 'digest' the neutral iodine with aromatic structures, resulting in a record $V_{\mathrm{oc}}$ deficit of $0.34 \mathrm{~V}$ and in a PCE of $21.4 \%$ for $\mathrm{p}-\mathrm{i}-\mathrm{n} \mathrm{PSCs}^{16}$. By using phenethylammonium iodide (PEAI), a major breakthrough in surface passivation (without thermal annealing) was achieved, resulting in

a record PCEs of $23.3 \%$ using a perovskite with a narrow bandgap $(1.53 \mathrm{eV})^{19}$. 
Impressively, the device showed an ERE of $8 \%$ and a $V_{\text {oc }}$ of $1.18 \mathrm{~V}, 94.4 \%$ of the SQ radiative limit. These improvements in device performance do not arise from the formation of a quasi-2D perovskite layer at the surface - as in other works that used $2 \mathrm{D}$ perovskites as the passivation layer - owing to the absence of a quasi-2D phase, and can thus be attributed to the use of PEAI.

A recent study has shown that passivation effects do not only depend on the functional groups used, but are also governed by the strength of the hydrogen bonds between the passivation functional groups and the perovskite ${ }^{129}$ (FIG. 4c). The hydrogen bonds between $\mathrm{FA}^{+}$and the amine groups of passivation molecules, for example, can be reduced by the presence of $\mathrm{O}$ atoms polarizing the passivation amine groups, owing to their electron-withdrawing nature. If a fluorine-containing polarized ferroelectric polymer is incorporated into a perovskite film, the fluorine can spontaneously form hydrogen bonds with $\mathrm{N}-\mathrm{H}$ in $\mathrm{MA}^{+}\left(\text {or } \mathrm{FA}^{+}\right)^{130}$. This strong interaction between polarized ferroelectric polymers and perovskite crystals can assist the perovskite arrangement under an external electric field, thus suppressing non-radiative recombination pathways.

As a result, molecules with various functional groups (such as Lewis acids and bases, metal-halide ions, ammonium groups, carboxyl and carboxylate groups, phosphate groups, sulfonic groups and aromatic structures) can neutralize or passivate defects in perovskites $^{16,19}$ (FIG. 4d). The passivation can happen through various mechanisms, including coordination bonds, ionic bonds and hydrogen bonds. The correlations between the typical functional groups and device performance improvements are provided in Supplementary Table 1. Lewis acids can passivate under-coordinated halides and $\mathrm{Pb}-\mathrm{I}$ antisite defects by forming a Lewis adduct by coordinate bonding, whereas under- 
coordinated lead ions and/or lead clusters can be reduced by Lewis bases that donate a lone pair of electrons ${ }^{56}$. A recent study has revealed that metal ions can heal negatively charged defects induced by A-site vacancies or undercoordinated halides at grain boundaries ${ }^{13}$. Ammonium cations can passivate negatively charged defects through electrostatic interactions, and the addition of halide ions can compensate halide vacancies. Other groups (such as the carboxylate, phosphate and sulfonic groups) can heal positively charged defects via ionic interactions. The carboxyl group is proposed for passivating negatively charged undercoordinated halides via hydrogen bonding, while its aromatic structures may reduce the number of acceptor-type defects induced by dissociative iodine ${ }^{16}$.

\section{[H2] Interface engineering}

Because most passivation molecules are dissolved into the perovskite solution or spin-coated onto the perovskite film, the resulting films exhibit variations in their surface chemistry and physics, which influence interface-induced recombination losses. For instance, by inserting an ultrathin polarized ferroelectric polymer layer at the perovskite/top extraction layer interface, a favorable energy-level alignment can be obtained, minimizing interface-induced recombination losses ${ }^{130}$. A recent work suggested a device architecture that use poly(3-hexylthiophene) as a hole-transport material without any dopants, and exploit a thin layer of wide-bandgap halide perovskite on top of the narrow-bandgap light-absorbing layer, facilitating carrier extraction and reduce backtransfer recombination losses at the top interface. Similarly, quasi-2D perovskites deposited on top of $3 \mathrm{D}$ perovskites can reduce the number of non-radiative recombination centers at the surface and grain boundaries. This layer, which has a wider bandgap than the 3D perovskite, also provides a spatial separation and an energy barrier that help to 
minimize interfacial recombination losses at the bulk perovskite/top extraction layer interface, yielding a low $V_{\text {oc }}$ deficit $(0.34 \mathrm{~V})$ and high PCE $(23.4 \%)^{131}$.

It is unclear whether such passivation strategies are effective for bottom interfaces, particularly for post-treatment passivation. For $\mathrm{TiO}_{2}$-based n-i-p PSCs, the losses at the bottom interfaces are generally mitigated using chlorinated $\mathrm{TiO}_{2}$ compact layers ${ }^{132}$. Inserting a thin interlayer between $\mathrm{SnO}_{2}$ and the perovskite, for example $\mathrm{PC}_{61} \mathrm{BM}$ :Poly(methyl methacrylate) (PMAA) composites, suppressed recombination losses at the interfacial contacts ${ }^{133-135}$. For $\mathrm{p}-\mathrm{i}-\mathrm{n}$ PSCs, adding a conjugated polyelectrolyte between the PTAA and the perovskite considerably reduced the interfacial recombination $\operatorname{losses}^{106}$. In-depth studies of passivation at the bottom interfaces are still quite limited.

\section{[H2] Formation of graded junctions}

Homojunctions and heterojunctions greatly influence the optoelectronic properties of defect-rich grain boundaries and surface regions, and can be exploited to improve device performance ${ }^{136,137}$. The semiconducting behaviour of perovskite absorbers is significantly influenced by the polarity of the substrate on which the perovskite crystallizes. For instance, perovskite absorbers crystallized on n-type substrates tend to be n-type in nature close to the interface, whereas near the p-interface they tend to be $p$-type in nature ${ }^{14}$. By exploiting the presence of defects, researchers realized a $\mathrm{p}$-n homojunction based on $\mathrm{MAPbI}_{3}$ peorvskites $^{138}$, in which the number of majority carriers and the charge carrier density were tuned through self-doping effects rather than extrinsic doping ${ }^{139,140}$. However, experimentally verifying whether the perovskite has p-type or n-type behavior, for example by Hall effect measurements, is extremely challenging owing to the presence of mobile ionic and electronic species, which can drift in electric fields and screen the Hall voltage, 
preventing it from building up. In some cases, changes in semiconductor type occur at the surface regions via conversion to a quasi-2D perovskite layer. Hall effect measurements generally reveal the average bulk properties, and cannot distinguish differences between bulk and surface regions. A potential solution is to probe the energy level profile using ultraviolet/inverse photoemission spectroscopy (UPS/IPES). However, the band bending and surface photovoltage at the perovskite surface need to be taken into consideration carefully. An alternative route is to use contactless ultrafast optical spectroscopy ${ }^{141}$, which is widely used in organic optoelectronics to probe charge or energy transfer processes across heterojunctions. This technique was also exploited to elucidate the changes in energy bands in surface regions relative to bulk regions ${ }^{141,142}$.

Recently, a solution-processed secondary growth technique was developed based on the use of guanidinium bromide (FIG. 5a), which is known to affect the semiconducting nature of perovskites ${ }^{21}$. In transient absorption measurements on quartz glass/perovskite/PMMA samples, a redshift of the ground-state bleach maxima from 730 to $750 \mathrm{~nm}$ was observed over the first few nanoseconds of exciting and probing the sample from the PMMA side, but not when the sample was excited and probed from the glass side (FIG. 5b). This suggests that a graded junction forms, with a wider bandgap close to the top surface, when samples are prepared using this process. Such changes resulted in an average $V_{\mathrm{oc}}$ improvement of $100 \mathrm{mV}$ in comparison to the control without a graded junction and in a certified PCE of $20.90 \%$ for p-i-n PSCs. The graded junction concept is also applicable to n-i-p PSCs based on inorganic perovskites and other perovskites ${ }^{143,144}$. Using n-hexyl trimethylammonium bromide, a thin layer of wide-bandgap halide perovskite can be grown on top of the narrow-bandgap absorbers $\left(E_{\mathrm{g}} \sim 1.53 \mathrm{eV}\right)$ in n-i-p PSCs; this double- 
layered halide architecture resulted in a certified PCE of $22.7 \%^{22}$. These PSCs exhibited good stability at $85 \%$ relative humidity without encapsulation (FIG. 5c), and a long-term operational stability of 1370 hours under 1-sun illumination at room temperature with encapsulation (FIG. 5d). However, we note that most works report long-term stability data based on only one or a few champion devices. In fact, a statistic analysis of the operational stability of a set of devices at the maximum power point under 1-sun illumination is needed to establish the reproducibility of the results ${ }^{145}$.

In these examples graded junctions, having a wider bandgap near the top surface, might reduce the total electron-hole recombination rate in this region of the sample, where defects are likely to exist, by making it favourable for one or both carriers to reside predominantly within the bulk of the perovskite absorbers, or away from the surface ${ }^{146}$. Graded junctions containing self-passivation molecules might mitigate or screen the defects throughout the thickness of the perovskite films.

Despite their contribution to the reduction of non-radiative recombination losses, precise control of graded junctions remains a challenge. In particular, the chemical compositions of top layers with wider bandgaps and their formation mechanism require further exploration.

\section{[H2] Feasibility of efficiencies exceeding 29\%}

The overall PCE of a solar cell is governed by the product of its $J_{\mathrm{sc}}, V_{\mathrm{oc}}$ and fill factor. A reduced $J_{\mathrm{sc}}$ mainly reflects optical losses and incomplete collections ${ }^{147}$, whereas reduced $V_{\mathrm{oc}}$ and fill factor suggest the presence of non-radiative recombination losses and resistance losses. Hence, light-absorption management and minimization of non-radiative 
recombination losses (at the surface and interface, and in the bulk) are effective means to overcome the present PCE limitations and to reach the radiative limit. Presently, the $V_{\text {oc }}$ deficit of a record-efficiency GaAs solar cells is $0.31 \mathrm{~V}^{148}$. Assuming a $V_{\text {oc }}$ deficit of 0.31 $\mathrm{V}$ (note that the present record $V_{\mathrm{oc}}$ deficit for PSCs is $0.34 \mathrm{~V}^{131}$ ) and an EQE approaching $95 \%$ (achievable if the optical losses are minimized) in a PSC, $J_{\text {sc }}$ can be calculated as a function of bandgap (see Supplementary Information for the calculating $J_{\mathrm{sc}}$ ) using the SQ theory (FIG. 6a). The $J_{s c}$ value for record-efficiency PSCs (corresponding to a bandgap of $1.53 \mathrm{eV}$ ) is $25.2 \mathrm{~mA} \mathrm{~cm}^{-2}$ without applying any light-trapping strategy ${ }^{19}$, reaching $90 \%$ of the SQ limit. The corresponding $\log (\mathrm{ERE})$ value is -0.66 . In Fig. 6b, the white dashed line represents the achievable $V_{\mathrm{oc}}$ as a function of bandgap with the $\log (\mathrm{ERE})$ value fixed at around -0.66. The achievable $V_{\mathrm{oc}}$ is $1.17 \mathrm{~V}$ for a bandgap of $1.48 \mathrm{eV}$ and $1.22 \mathrm{~V}$ for a bandgap of $1.53 \mathrm{eV}$. The fill factors and PCEs were also calculated (FIG. 6c,d). Notably, owing to the suppression of non-radiative recombination, the calculated fill factors of two state-of-the-art perovskites approach 0.90, delivering potential PCEs of over $29 \%$. However, beyond non-radiative recombination losses, the fill factors of solar cells can also be affected by the parasitic resistance. Controlling the parasitic losses resulting from the additional resistance of the charge extraction layers has enabled the fill factor to approach the radiative limit ${ }^{149}$. This result offers the possibility of avoiding charge transport limitations to push the fill factor towards the radiative limit.

Thus, minimizing non-radiative recombination losses and optical losses is the next step for realizing high-efficiency single-junction PSCs. Moreover, although in tandem cells (such as perovskite-on-silicon, perovskite-on-CIGS and all-perovskite tandem cells) reducing non-radiative recombination losses and optical losses is in principle very complex, 
the lessons learned from single-junction PSCs should push forward also this fastdeveloping and exciting field.

\section{[H1] Future perspectives}

We have systematically rationalized the scientific and technological efforts undertaken to understand the origin of non-radiative recombination losses, and various means to mitigate them in complete PSCs. However, we are still at the early stages of understanding and addressing the challenges related to the production of defect-free PSCs for practical applications. Substantial efforts will be needed to minimize non-radiative recombination losses and push PCEs towards the SQ limit.

Defect capture and mismatched energy level alignment at the interfaces are the predominant sources of non-radiative recombination losses. To address defect-assisted recombination losses, a reliable and high-throughput method to calculate the electronphonon interactions and defect capture cross sections needs to be developed. Quantifying the defect capture cross sections allows us to assess the accuracy of the defect density determined by experiments, and will help to understand the defect chemistry. Normally, defect capture events (non-radiative recombination) occur when the electrostatic potential energy from a charged defect exceeds the thermal energy, and the process is influenced by the dielectric constant of the perovskite through the screening effect. In this context, the density of defects, capture cross section, depth of defects and drift velocity of carriers should be considered as a whole in future work. In addition, the energy level alignment plays a key role in determining the $V_{\mathrm{oc}}$ and PCE. However, the physical principles governing the energy level alignment at the perovskite/charge extraction layer interface remain unknown, because the metastable nature of perovskite materials, combined with the 
diverse solution-based methods used in materials fabrications, makes it difficult to establish a universal master equation to compute the energy offsets. The next key step is to explore the interfacial energy level alignment associated with point defects, surface states, ion migration and chemical reaction and diffusion, and to understand how recombination losses depend on the energy alignment.

Eliminating the defects at the perovskite surfaces and grain boundaries remains a key challenge. Advanced processing techniques can be explored to achieve this target. Epitaxial growth is a feasible approach to prepare defect-free crystals, and has been widely used for fabricating high-performance semiconductor devices. Perovskites with a low density of defects are routinely obtained with epitaxial growth ${ }^{150}$ by tuning the composition and deposition conditions. Interestingly, epitaxial growth of perovskites thin films can be achieved on a variety of single-crystal and single-crystal-like substrates simply by spin coating ${ }^{151}$. Transferring this technique to the large-scale production of PSCs requires more research in process engineering because of changes in the materials and underlying substrates used. Heteroepitaxial growth may be used for single-crystal perovskite devices, whose performance is still inferior to that of polycrystalline perovskite PSCs despite the lower density of defects in single-crystal perovskites, which is possibly due to the non-ideal electrical contacts.

Because perovskite quantum dots (QDs) display a single-crystal-like crystallographic structure and quantum confinement effect, it should be possible to use them to minimize the defects in solid perovskite films via ligand engineering. We envisage that high-quality single-crystal-like perovskite films will be realized through the in-situ conversion of the QDs after the complete removal of ligands. Thus, PSCs based on 
perovskite QDs or single crystals are worth exploring.

Although a large variety of passivation molecules have been tested, the library of available molecules is still relatively limited. A pressing issue in the development of new passivation molecules is to understand what kind of functional groups play the most important role, and what are the potential targets for passivation. To resolve this issue, the origin of the defects in the perovskite films processed using various precursor compositions and methods needs to be identified. High-throughput experiments and machine-learning techniques have become increasingly accessible, and may accelerate the experimental cycles for the development of new passivation materials. The feasibility of accelerating perovskite-inspired materials production has been recently demonstrated using a combination of high-throughput synthesis and machine-learning-assisted data diagnostics of lead-halide and lead-free perovskites (75 compositions) within a 2 -month period ${ }^{152}$ in a fully automated laboratory for materials discovery and development, more than 10 times faster than human analysis and with 90\% accuracy. Additionally, developing 3D fluorescence imaging of individual grains in perovskite thin films is promising to monitor the passivation processes, uncovering the passivation mechanism of all types of defects.

Creating stable graded junctions in PSCs can reduce the carrier recombination rate at heterojunction interfaces. However, the precise energy level shifts between the bottom interfaces and the top surface regions are not yet clear. A precise control of the graded junction might be achieved with alternative fabrication methods, such as spray coating or physical vapour deposition. There is also an urgent need to go beyond the present material library to fine-tune the graded junction. To this end, machine learning might serve as a powerful tool, providing a comprehensive analysis from materials to device 
performance ${ }^{152,153}$. The prospect of creating stable graded junction based on new materials may stimulate fundamental studies of new physics and chemistry, and result in better control of defects within perovskite films. For example, the optical losses at the bottom interfaces might be reduced by introducing an optically tuned junction. Currently, research on the buried bottom interfaces is rarely reported, and more work is needed to fully uncover the properties of these interfaces, including their surface chemistry and defect physics, and to quantify their contributions to non-radiative recombination losses.

Lastly, advanced photovoltaic research on topics such as tandem architectures ${ }^{85}$, singlet fission ${ }^{154}$ and hot carriers ${ }^{38}$ all require an in-depth understanding of the origin and nature of non-radiative recombination losses. Regarding PSCs, on the basis of future advances in both theory and experiments, we envisage that their PCEs will exceed those of crystalline silicon solar cells in the next few years, making them competitive in the commercial photovoltaic market. 


\section{References}

1. Liu, M., Johnston, M. B. \& Snaith, H. J. Efficient planar heterojunction perovskite solar cells by vapour deposition. Nature 501, 395 (2013).

2. Wang, L. et al. A $\mathrm{Eu}^{3+}-\mathrm{Eu}^{2+}$ ion redox shuttle imparts operational durability to $\mathrm{Pb}-\mathrm{I}$ perovskite solar cells. Science 363, 265-270 (2019).

3. Kojima, A., Teshima, K., Shirai, Y. \& Miyasaka, T. Organometal halide perovskites as visible-light sensitizers for photovoltaic cells. J. Am. Chem. Soc. 131, 6050-6051 (2009).

4. Jeon, N. J. et al. A fluorene-terminated hole-transporting material for highly efficient and stable perovskite solar cells. Nat. Energy 3, 682-689 (2018).

5. National Renewable Energy Laboratory, Best research-cell efficiencies chart (2019); www.nrel.gov/pv/assets/pdfs/bestresearch-cell-efficiencies.20190703.pdf

6. Tong, J. et al. Carrier lifetimes of $>1 \mu \mathrm{s}$ in $\mathrm{Sn}-\mathrm{Pb}$ perovskites enable efficient allperovskite tandem solar cells. Science 364, 475-479 (2019).

7. Nayak, P. K., Mahesh, S., Snaith, H. J. \& Cahen, D. Photovoltaic solar cell technologies: Analysing the state of the art. Nat. Rev. Mater. 4, 269-285 (2019).

8. Chen, J. \& Park, N.-G. Causes and solutions of recombination in perovskite solar cells. Adv. Mater. 1803019 (2018).

9. Aydin, E., De Bastiani, M. \& De Wolf, S. Defect and contact passivation for perovskite solar cells. Adv. Mater. 1900428 (2019).

10. Liu, Y. et al. Ultrahydrophobic 3D/2D fluoroarene bilayer-based water-resistant perovskite solar cells with efficiencies exceeding 22\%. Sci. Adv. 5, eaaw2543 (2019).

11. Sarritzu, V. et al. Optical determination of shockley-read-hall and interface recombination currents in hybrid perovskites. Sci. Rep. 7, 44629 (2017).

12. Tvingstedt, K. et al. Radiative efficiency of lead iodide based perovskite solar cells. Sci. Rep. 4, 6071 (2014).

13. Abdi-Jalebi, M. et al. Maximizing and stabilizing luminescence from halide perovskites with potassium passivation. Nature 555, 497 (2018).

14. Saliba, M. et al. Incorporation of rubidium cations into perovskite solar cells improves photovoltaic performance. Science 354, 206-209 (2016).

15. Shao, Y., Xiao, Z., Bi, C., Yuan, Y. \& Huang, J. Origin and elimination of photocurrent hysteresis by fullerene passivation in $\mathrm{CH}_{3} \mathrm{NH}_{3} \mathrm{PbI}_{3}$ planar heterojunction solar cells. Nat. Commun. 5, 5784 (2014).

16. Yang, S. et al. Tailoring passivation molecular structures for extremely small opencircuit voltage loss in perovskite solar cells. J. Am. Chem. Soc. 141, 5781-5787 (2019).

17. Wang, Q., Dong, Q., Li, T., Gruverman, A. \& Huang, J. Thin insulating tunneling contacts for efficient and water-resistant perovskite solar cells. Adv. Mater. 28, 67346739 (2016).

18. Shao, Y., Yuan, Y. \& Huang, J. Correlation of energy disorder and open-circuit voltage in hybrid perovskite solar cells. Nat. Energy 1, 15001 (2016).

19. Jiang, Q. et al. Surface passivation of perovskite film for efficient solar cells. Nat. Photon. 13, 460-466 (2019).

20. Zheng, X. et al. Defect passivation in hybrid perovskite solar cells using quaternary ammonium halide anions and cations. Nat. Energy 2, 17102 (2017). 
21. Luo, D. et al. Enhanced photovoltage for inverted planar heterojunction perovskite solar cells. Science 360, 1442-1446 (2018).

22. Jung, E. H. et al. Efficient, stable and scalable perovskite solar cells using poly(3hexylthiophene). Nature 567, 511-515 (2019).

23. Wu, S. et al. Efficient large guanidinium mixed perovskite solar cells with enhanced photovoltage and low energy losses. Chem. Commun. 55, 4315-4318 (2019).

24. Zhou, W. et al. Zwitterion coordination induced highly orientational order of $\mathrm{CH}_{3} \mathrm{NH}_{3} \mathrm{PbI}_{3}$ perovskite film delivers a high open circuit voltage exceeding $1.2 \mathrm{~V}$. Adv. Funct. Mater. 29, 1901026 (2019).

25. Rong, Y. et al. Challenges for commercializing perovskite solar cells. Science 361, eaat8235 (2018).

26. Lee, M. M., Teuscher, J., Miyasaka, T., Murakami, T. N. \& Snaith, H. J. Efficient hybrid solar cells based on meso-superstructured organometal halide perovskites. Science 338, 643-647 (2012).

27. Lin, Y. et al. П-conjugated lewis base: Efficient trap-passivation and charge-extraction for hybrid perovskite solar cells. Adv. Mater. 29, 1604545 (2017).

28. Chen, W. et al. Efficient and stable large-area perovskite solar cells with inorganic charge extraction layers. Science 350, 944-948 (2015).

29. Luo, D. et al. Dual-source precursor approach for highly efficient inverted planar heterojunction perovskite solar cells. Adv. Mater. 29, 1604758 (2017).

30. Krogstrup, P. et al. Single-nanowire solar cells beyond the Shockley-Queisser limit. Nat. Photon. 7, 306 (2013).

31 Sha, W. E. I., Ren, X., Chen, L. \& Choy, W. C. H. The efficiency limit of $\mathrm{CH}_{3} \mathrm{NH}_{3} \mathrm{PbI}_{3}$ perovskite solar cells. Appl. Phys. Lett. 106, 221104 (2015).

32. Stranks, S. D., Hoye, R. L. Z., Di, D., Friend, R. H. \& Deschler, F. The physics of light emission in halide perovskite devices. Adv. Mater. 1803336 (2019).

33. Hutter, E. M. et al. Direct-indirect character of the bandgap in methylammonium lead iodide perovskite. Nat. Mater. 16, 115 (2016).

34. Stranks, S. D. \& Plochocka, P. The influence of the rashba effect. Nat. Mater. 17, 381382 (2018).

35. Stranks, S. D. Nonradiative losses in metal halide perovskites. ACS Energy Lett. 2, 1515-1525 (2017).

36. Eperon, G. E., Hörantner, M. T. \& Snaith, H. J. Metal halide perovskite tandem and multiple-junction photovoltaics. Nat. Rev. Chem. 1, 0095 (2017).

37. Filipič, M. et al. $\mathrm{CH}_{3} \mathrm{NH}_{3} \mathrm{PbI}_{3}$ perovskite/silicon tandem solar cells: Characterization based optical simulations. Opt. Express 23, A263-A278 (2015).

38. Guo, Z. et al. Long-range hot-carrier transport in hybrid perovskites visualized by ultrafast microscopy. Science 356, 59-62 (2017).

39. Yang, Y. et al. Observation of a hot-phonon bottleneck in lead-iodide perovskites. Nat. Photon. 10, 53 (2015).

40. Fu, J. et al. Hot carrier cooling mechanisms in halide perovskites. Nat. Commun. 8, 1300 (2017).

41. Zhu, H. et al. Screening in crystalline liquids protects energetic carriers in hybrid perovskites. Science 353, 1409-1413 (2016).

42. Quan, L. N., García de Arquer, F. P., Sabatini, R. P. \& Sargent, E. H. Perovskites for light emission. Adv. Mater. 30, 1801996 (2018). 
43. Richter, J. M. et al. Ultrafast carrier thermalization in lead iodide perovskite probed with two-dimensional electronic spectroscopy. Nat. Commum. 8, 376 (2017).

44. Price, M. B. et al. Hot-carrier cooling and photoinduced refractive index changes in organic-inorganic lead halide perovskites. Nat. Commun. 6, 8420 (2015).

45. Bretschneider, S. A. et al. Quantifying polaron formation and charge carrier cooling in lead-iodide perovskites. Adv. Mater. 30, 1707312 (2018).

46. Joshi, P. P., Maehrlein, S. F. \& Zhu, X. Dynamic screening and slow cooling of hot carriers in lead halide perovskites. Adv. Mater. 1803054 (2019).

47. Ball, J. M. \& Petrozza, A. Defects in perovskite-halides and their effects in solar cells. Nat. Energy 1, 16149 (2016).

48. Huang, J., Yuan, Y., Shao, Y. \& Yan, Y. Understanding the physical properties of hybrid perovskites for photovoltaic applications. Nat. Rev. Mater. 2, 17042 (2017).

49. de Quilettes, D. W. et al. Impact of microstructure on local carrier lifetime in perovskite solar cells. Science 348, 683-686 (2015).

50. Wright, A. D. et al. Electron-phonon coupling in hybrid lead halide perovskites. Nat. Commum. 7, 11755 (2016).

51. Ran, C., Xu, J., Gao, W., Huang, C. \& Dou, S. Defects in metal triiodide perovskite materials towards high-performance solar cells: Origin, impact, characterization, and engineering. Chem. Soc. Rev. 47, 4581-4610 (2018).

52. Hill, A. H., Kennedy, C. L., Massaro, E. S. \& Grumstrup, E. M. Perovskite carrier transport: Disentangling the impacts of effective mass and scattering time through microscopic optical detection. J. Phys. Chem. Lett. 9, 2808-2813 (2018).

53. Wang, Z. et al. High irradiance performance of metal halide perovskites for concentrator photovoltaics. Nat. Energy 3, 855-861 (2018).

54. Jones, T. W. et al. Lattice strain causes non-radiative losses in halide perovskites. Energy Environ. Sci. 12, 596-606 (2019).

55. Li, L. et al. The additive coordination effect on hybrids perovskite crystallization and high-performance solar cell. Adv. Mater. 28, 9862-9868 (2016).

56. Chen, B., Rudd, P. N., Yang, S., Yuan, Y. \& Huang, J. Imperfections and their passivation in halide perovskite solar cells. Chem. Soc. Rev. 48, 3842-3867 (2019).

57. Yin, W.-J., Shi, T. \& Yan, Y. Unusual defect physics in $\mathrm{CH}_{3} \mathrm{NH}_{3} \mathrm{PbI}_{3}$ perovskite solar cell absorber. Appl. Phys. Lett. 104, 063903 (2014).

58. Solanki, A. et al. Cation influence on carrier dynamics in perovskite solar cells. Nano Energy 58, 604-611 (2019).

59. He, Y. \& Galli, G. Perovskites for solar thermoelectric applications: A first principle study of $\mathrm{CH}_{3} \mathrm{NH}_{3} \mathrm{AI}_{3}(\mathrm{~A}=\mathrm{Pb}$ and $\mathrm{Sn})$. Chem. Mater. 26, 5394-5400 (2014).

60. Wu, N. et al. Identifying the cause of voltage and fill factor losses in perovskite solar cells by using luminescence measurements. Energy Technol. 5, 1827-1835 (2017).

61. Bardeen, J. Surface states and rectification at metal semi-conductor contact. Phys. Rev. 71, 717-727 (1947).

62. Adinolfi, V. et al. The in-gap electronic state spectrum of methylammonium lead iodide single-crystal perovskites. Adv. Mater. 28, 3406-3410 (2016).

63. Aranda, C., Guerrero, A. \& Bisquert, J. Ionic effect enhances light emission and the photovoltage of methylammonium lead bromide perovskite solar cells by reduced surface recombination. ACS Energy Lett. 4, 741-746 (2019). 
64. Yang, D. et al. Stable efficiency exceeding $20.6 \%$ for inverted perovskite solar cells through polymer-optimized PCBM electron-transport layers. Nano Lett. (2019).

65. Abdi-Jalebi, M. et al. Charge extraction via graded doping of hole transport layers gives highly luminescent and stable metal halide perovskite devices. Sci. Adv. 5, eaav2012 (2019).

66. Halvani Anaraki, E. et al. Low-temperature $\mathrm{Nb}$-doped $\mathrm{SnO}_{2}$ electron-selective contact yields over $20 \%$ efficiency in planar perovskite solar cells. ACS Energy Lett. 3, 773778 (2018).

67. Arora, N. et al. Perovskite solar cells with $\mathrm{CuSCN}$ hole extraction layers yield stabilized efficiencies greater than 20\%. Science 358, 768-771 (2017).

68. Tress, W., Leo, K. \& Riede, M. Optimum mobility, contact properties, and opencircuit voltage of organic solar cells: A drift-diffusion simulation study. Phys. Rev. B 85, 155201 (2012).

69. Tress, W. et al. Interpretation and evolution of open-circuit voltage, recombination, ideality factor and subgap defect states during reversible light-soaking and irreversible degradation of perovskite solar cells. Energy Environ. Sci. 11, 151-165 (2018).

70. Wolff, C. M. et al. Reduced interface-mediated recombination for high open-circuit voltages in $\mathrm{CN}_{3} \mathrm{NH}_{3} \mathrm{PbI}_{3}$ solar cells. Adv. Mater. 29, 1700159 (2017).

71. Wang, S. et al. Large guanidinium cation enhance photovoltage for perovskite solar cells via solution-processed secondary growth technique. Sol. Energy 176, 118-125 (2018).

72. Stolterfoht, M. et al. The impact of energy alignment and interfacial recombination on the internal and external open-circuit voltage of perovskite solar cells. Energy Environ. Sci. (2019).

73. Wang, J. et al. Reducing surface recombination velocities at the electrical contacts will improve perovskite photovoltaics. ACS Energy Lett. 4, 222-227 (2019).

74. Bi, C. et al. Non-wetting surface-driven high-aspect-ratio crystalline grain growth for efficient hybrid perovskite solar cells. Nat. Commun. 6, 7747 (2015).

75. Jiang, Q. et al. Enhanced electron extraction using $\mathrm{SnO}_{2}$ for high-efficiency planarstructure $\mathrm{HC}\left(\mathrm{NH}_{2}\right)_{2} \mathrm{PbI}_{3}$-based perovskite solar cells. Nat. Energy 2, 16177 (2016).

76. Hou, Y. et al. A generic interface to reduce the efficiency-stability-cost gap of perovskite solar cells. Science 358, 1192-1197 (2017).

77. Evans, T. J. S. et al. Competition between hot-electron cooling and large polaron screening in $\mathrm{CsPbBr}_{3}$ perovskite single crystals. J. Phys. Chem. C 122, 13724-13730 (2018).

78. Miyata, K., Atallah, T. L. \& Zhu, X.-Y. Lead halide perovskites: Crystal-liquid duality, phonon glass electron crystals, and large polaron formation. Sci. Adv. 3, e1701469 (2017).

79. Guo, Z., Wu, X., Zhu, T., Zhu, X. \& Huang, L. Electron-phonon scattering in atomically thin 2D perovskites. ACS Nano 10, 9992-9998 (2016).

80. Motta, C. \& Sanvito, S. Electron-phonon coupling and polaron mobility in hybrid perovskites from first principles. J. Phys. Chem. C 122, 1361-1366 (2018).

81. Karakus, M. et al. Phonon-electron scattering limits free charge mobility in methylammonium lead iodide perovskites. J. Phys. Chem. Lett. 6, 4991-4996 (2015).

82. Gong, X. et al. Electron-phonon interaction in efficient perovskite blue emitters. Nat. Mater. 17, 550-556 (2018). 
83. Wright, A. D. et al. Band-tail recombination in hybrid lead iodide perovskite. Adv. Funct. Mater. 27, 1700860 (2017).

84. De Wolf, S. et al. Organometallic halide perovskites: Sharp optical absorption edge and its relation to photovoltaic performance. J. Phys. Chem. Lett. 5, 1035-1039 (2014).

85. McMeekin, D. P. et al. A mixed-cation lead mixed-halide perovskite absorber for tandem solar cells. Science 351, 151-155 (2016).

86. Rau, U., Blank, B., Müller, T. C. M. \& Kirchartz, T. Efficiency potential of photovoltaic materials and devices unveiled by detailed-balance analysis. Phys. Rev. Appl. 7, 044016 (2017).

87. Snaith, H. J. Present status and future prospects of perovskite photovoltaics. Nat. Mater. 17, 372-376 (2018).

88. Rau, U. Reciprocity relation between photovoltaic quantum efficiency and electroluminescent emission of solar cells. Phys. Rev. B 76, 085303 (2007).

89. Green, M. A. Radiative efficiency of state-of-the-art photovoltaic cells. Prog. Photovolt: Res. Appl. 20, 472-476 (2012).

90. Pazos-Outon, L. M., Xiao, T. P. \& Yablonovitch, E. Fundamental efficiency limit of lead iodide perovskite solar cells. J. Phys. Chem. Lett. 9, 1703-1711 (2018).

91. Tress, W. et al. Predicting the open-circuit voltage of $\mathrm{CH}_{3} \mathrm{NH}_{3} \mathrm{PbI}_{3}$ perovskite solar cells using electroluminescence and photovoltaic quantum efficiency spectra: The role of radiative and non-radiative recombination. Adv. Energy Mater. 5, 1400812 (2015).

92. Shi, X.-B. et al. Optical energy losses in organic-inorganic hybrid perovskite lightemitting diodes. Adv. Opt. Mater. 6, 1800667 (2018).

93. Tress, W. Perovskite solar cells on the way to their radiative efficiency limit - insights into a success story of high open-circuit voltage and low recombination. Adv. Energy Mater. 7, 1602358 (2017).

94. Yoshikawa, K. et al. Silicon heterojunction solar cell with interdigitated back contacts for a photoconversion efficiency over 26\%. Nat. Energy 2, 17032 (2017).

95. Green, M. A. \& Bremner, S. P. Energy conversion approaches and materials for highefficiency photovoltaics. Nat. Mater. 16, 23 (2016).

96. Quan, L. N. et al. Tailoring the energy landscape in quasi-2D halide perovskites enables efficient green-light emission. Nano Lett. 17, 3701-3709 (2017).

97. Xiao, Z. et al. Giant switchable photovoltaic effect in organometal trihalide perovskite devices. Nat. Mater. 14, 193 (2014).

98. Birkhold, S. T. et al. Interplay of mobile ions and injected carriers creates recombination centers in metal halide perovskites under bias. ACS Energy Lett. 3, 1279-1286 (2018).

99. Reislöhner, U., Metzner, H. \& Ronning, C. Hopping conduction observed in thermal admittance spectroscopy. Phys. Rev. Lett. 104, 226403 (2010).

100.Losee, D. L. Admittance spectroscopy of impurity levels in schottky barriers. J. Appl. Phys. 46, 2204-2214 (1975).

101.Wang, S., Kaienburg, P., Klingebiel, B., Schillings, D. \& Kirchartz, T. Understanding thermal admittance spectroscopy in low-mobility semiconductors. J. Phys. Chem. C 122, 9795-9803 (2018).

102.Saba, M. et al. Correlated electron-hole plasma in organometal perovskites. Nat. Commun. 5, 5049 (2014). 
103. Yang, W. S. et al. Iodide management in formamidinium-lead-halide-based perovskite layers for efficient solar cells. Science 356, 1376-1379 (2017).

104.Ono, L. K. \& Qi, Y. Surface and interface aspects of organometal halide perovskite materials and solar cells. J. Phys. Chem. Lett. 7, 4764-4794 (2016).

105.Ohmann, R. et al. Real-space imaging of the atomic structure of organic-inorganic perovskite. J. Am. Chem. Soc. 137, 16049-16054 (2015).

106.Stolterfoht, M. et al. Visualization and suppression of interfacial recombination for high-efficiency large-area pin perovskite solar cells. Nat. Energy 3, 847-854 (2018).

107.El-Hajje, G. et al. Quantification of spatial inhomogeneity in perovskite solar cells by hyperspectral luminescence imaging. Energy Environ. Sci. 9, 2286-2294 (2016).

108.Jeon, N. J. et al. Solvent engineering for high-performance inorganic-organic hybrid perovskite solar cells. Nat. Mater. 13, 897 (2014).

109.Tu, Y. et al. Diboron-assisted interfacial defect control strategy for highly efficient planar perovskite solar cells. Adv. Mater. 30, 1805085 (2018).

110.Liang, P. W. et al. Additive enhanced crystallization of solution-processed perovskite for highly efficient planar-heterojunction solar cells. Adv. Mater. 26, 3748-3754 (2014).

111.Cao, Y. et al. Perovskite light-emitting diodes based on spontaneously formed submicrometre-scale structures. Nature 562, 249-253 (2018).

112.Lin, K. et al. Perovskite light-emitting diodes with external quantum efficiency exceeding 20 per cent. Nature 562, 245-248 (2018).

113.Zhang, L. et al. Ultra-bright and highly efficient inorganic based perovskite lightemitting diodes. Nat. Commun. 8, 15640 (2017).

114.Kim, M. et al. Methylammonium chloride induces intermediate phase stabilization for efficient perovskite solar cells. Joule (2019).

115.Ban, M. et al. Solution-processed perovskite light emitting diodes with efficiency exceeding $15 \%$ through additive-controlled nanostructure tailoring. Nat. Commun. 9, 3892 (2018).

116.Yang, W. S. et al. High-performance photovoltaic perovskite layers fabricated through intramolecular exchange. Science 348, 1234-1237 (2015).

117.Jiang, Q. et al. Planar-structure perovskite solar cells with efficiency beyond $21 \%$. Adv. Mater. 29, 1703852 (2017).

118.Yang, R. et al. Oriented quasi-2D perovskites for high performance optoelectronic devices. Adv. Mater. 30, 1804771 (2018).

119.Jeon, N. J. et al. Compositional engineering of perovskite materials for highperformance solar cells. Nature 517, 476 (2015).

120.Chao, L. et al. Room-temperature molten salt for facile fabrication of efficient and stable perovskite solar cells in ambient air. Chem 5, 995-1006 (2019).

121.Hu, Q. et al. In situ dynamic observations of perovskite crystallisation and microstructure evolution intermediated from $\left[\mathrm{PbI}_{6}\right]^{4-}$ cage nanoparticles. Nat. Commum. 8, 15688 (2017).

122.Brenes, R., Eames, C., Bulović, V., Islam, M. S. \& Stranks, S. D. The impact of atmosphere on the local luminescence properties of metal halide perovskite grains. Adv. Mater. 30, 1706208 (2018). 
123.Abdi-Jalebi, M. et al. Potassium- and rubidium-passivated alloyed perovskite films: Optoelectronic properties and moisture stability. ACS Energy Lett. 3, 2671-2678 (2018).

124.Correa-Baena, J.-P. et al. Homogenized halides and alkali cation segregation in alloyed organic-inorganic perovskites. Science 363, 627-631 (2019).

125.Kubicki, D. J. et al. Phase segregation in Cs-, $\mathrm{Rb}$ - and K-doped mixed-cation $(\mathrm{MA})_{\mathrm{x}}(\mathrm{FA})_{1-\mathrm{x}} \mathrm{PbI}_{3}$ hybrid perovskites from solid-state NMR. J. Am. Chem. Soc. 139, 14173-14180 (2017).

126.Kuai, L. et al. Passivating crystal boundaries with potassium-rich phase in organic halide perovskite. Solar RRL 3, 1900053 (2019).

127.Li, N. et al. Cation and anion immobilization through chemical bonding enhancement with fluorides for stable halide perovskite solar cells. Nat. Energy 4, 408-415 (2019).

128.Liu, T. et al. Stable formamidinium-based perovskite solar cells via in situ grain encapsulation. Adv. Energy Mater. 8, 1800232 (2018).

129.Xu, W. et al. Rational molecular passivation for high-performance perovskite lightemitting diodes. Nat. Photon. 13, 418-424 (2019).

130.Zhang, C. C. et al. Polarized ferroelectric polymers for high-performance perovskite solar cells. Adv. Mater. 31, 1902222 (2019).

131.Yoo, J. J. et al. An interface stabilized perovskite solar cell with high stabilized efficiency and low voltage loss. Energy Environ. Sci. 12, 2192-2199 (2019).

132. Tan, H. et al. Efficient and stable solution-processed planar perovskite solar cells via contact passivation. Science 355, 722-726 (2017).

133.Peng, J. et al. Interface passivation using ultrathin polymer-fullerene films for highefficiency perovskite solar cells with negligible hysteresis. Energy Environ. Sci. 10, 1792-1800 (2017).

134.Peng, J. et al. A universal double-side passivation for high open-circuit voltage in perovskite solar cells: Role of carbonyl groups in poly(methyl methacrylate). $A d v$. Energy Mater. 8, 1801208 (2018).

135.Turren-Cruz, S. H., Hagfeldt, A. \& Saliba, M. Methylammonium-free, highperformance, and stable perovskite solar cells on a planar architecture. Science 362, 449-453 (2018).

136.Masuko, K. et al. Achievement of more than $25 \%$ conversion efficiency with crystalline silicon heterojunction solar cell. IEEE J. Photovolt. 4, 1433-1435 (2014).

137.Wu, Y. et al. Monolithic perovskite/silicon-homojunction tandem solar cell with over 22\% efficiency. Energy Environ. Sci. 10, 2472-2479 (2017).

138.Cui, P. et al. Planar p-n homojunction perovskite solar cells with efficiency exceeding 21.3\%. Nat. Energy 4, 150-159 (2019).

139.Wang, Q. et al. Qualifying composition dependent $\mathrm{p}$ and $\mathrm{n}$ self-doping in $\mathrm{CH}_{3} \mathrm{NH}_{3} \mathrm{PbI}_{3}$. Appl. Phys. Lett. 105, 163508 (2014).

140. Yuan, Y. et al. Anomalous photovoltaic effect in organic-inorganic hybrid perovskite solar cells. Sci. Adv. 3, e1602164 (2017).

141.Bakulin, A. A. et al. The role of driving energy and delocalized states for charge separation in organic semiconductors. Science 335, 1340-1344 (2012).

142. Yuan, M. et al. Perovskite energy funnels for efficient light-emitting diodes. Nat. Nanotechnol. 11, 872 (2016). 
143.Bian, H. et al. Graded bandgap $\mathrm{CsPbI}_{2+\mathrm{x}} \mathrm{Br}_{1-\mathrm{x}}$ perovskite solar cells with a stabilized efficiency of 14.4\%. Joule 2, 1500-1510 (2018).

144.Cho, K. T. et al. Highly efficient perovskite solar cells with a compositionally engineered perovskite/hole transporting material interface. Energy Environ. Sci. 10, 621-627 (2017).

145.Bai, S. et al. Planar perovskite solar cells with long-term stability using ionic liquid additives. Nature 571, 245-250 (2019).

146.Chirilă, A. et al. Highly efficient $\mathrm{Cu}(\mathrm{In}, \mathrm{Ga}) \mathrm{Se}_{2}$ solar cells grown on flexible polymer films. Nat. Mater. 10, 857 (2011).

147.Polman, A., Knight, M., Garnett, E. C., Ehrler, B. \& Sinke, W. C. Photovoltaic materials: Present efficiencies and future challenges. Science 352, aad4424 (2016).

148.Green, M. A. et al. Solar cell efficiency tables (version 52). Prog. Photovolt: Res. Appl. 26, 427-436 (2018).

149.Stolterfoht, M. et al. Approaching the fill factor Shockley-Queisser limit in stable, dopant-free triple cation perovskite solar cells. Energy Environ. Sci. 10, 1530-1539 (2017).

150.Chen, J. et al. Vapor-phase epitaxial growth of aligned nanowire networks of cesium lead halide perovskites $\left(\mathrm{CsPbX}_{3}, \mathrm{X}=\mathrm{Cl}, \mathrm{Br}, \mathrm{I}\right)$. Nano Lett. 17, 460-466 (2017).

151.Kelso, M. V., Mahenderkar, N. K., Chen, Q., Tubbesing, J. Z. \& Switzer, J. A. Spin coating epitaxial films. Science 364, 166-169 (2019).

152.Sun, S. et al. Accelerated development of perovskite-inspired materials via highthroughput synthesis and machine-learning diagnosis. Joule 3, 1437-1451 (2019).

153.Odabaş1, Ç. \& Yıldırım, R. Performance analysis of perovskite solar cells in 20132018 using machine-learning tools. Nano Energy 56, 770-791 (2019).

154.Lu, H., Chen, X., Anthony, J. E., Johnson, J. C. \& Beard, M. C. Sensitizing singlet fission with perovskite nanocrystals. J. Am. Chem. Soc. 141, 4919-4927 (2019).

\section{Acknowledgments}

This work was funded by the 973 Program of China (2015CB932203), the National Natural

Science Foundation of China (91733301, 61722501, and 61377025), W. Z. thanks EPSRC

New Investigator Award (2018; EP/R043272/1) for financial support.

\section{Author contributions}

All authors contributed to the discussion of content. D.L. and R.S. researched most of the data and wrote the draft. W.Z., Q.G. and R.Z. revised the manuscript before submission.

\section{Competing financial interests}

The authors declare no competing interests. 


\section{Supplementary information}

Supplementary information is available for this paper.

Figure 1| Perovskite solar cell configurations and record cell parameters compared to the Shockley-Queissier limit. a| Illustration of a n-i-p perovskite solar cell (PSC) with a mesoscopic layer, of a regular n-i-p planar heterojunction PSC, and of a p-i-n planar heterojunction PSC (also known as inverted planar PSC). b| Power conversion efficiency (PCE) limit as a function of bandgap for single-junction solar cells (dashed line) calculated using the Shockley-Queissier (SQ) theory. The record efficiencies of several photovoltaic materials (REF. 148) are also shown (triangles). c| Plot of calculated open-circuit voltage, $V_{\mathrm{oc}}$, at the radiative limit, $V_{\mathrm{oc}}$, rad, as a function of bandgap (dashed line), and $V_{\mathrm{oc}}$ of devices with record PCEs (triangles). The red and blue dots represent the record $V_{\text {oc }}$ of PSCs with n-i-p (REF. 19) and p-i-n (REF. 21) structures, respectively. The $V_{\mathrm{g}}=E_{\mathrm{g}} / \mathrm{q}$ line is shown for reference (solid line), and the voltage gap $\left(V_{g}-V_{\mathrm{oc}}\right.$, rad $)$ is indicated by the arrow.

Figure 2| Charge-carrier generation and recombination kinetics. a| Fundamental light excitation processes in perovskite absorbers. $E_{\mathrm{c}}$ and $E_{\mathrm{v}}$ are the conduction band minimum and valence band maximum, respectively. $\mathbf{b} \mid$ Generalized recombination dynamics of the photo-generated carriers, including radiative, defect-assisted, and direct and indirect Auger recombination. c| Diagrams showing the origins of interface-induced recombination losses, including deep-level defects within the perovskite absorber (type I), unfavorable band alignment at the interface and defects in the perovskite (type II), back-transfer-induced recombination (type III), and defect-assisted recombination in the electron extraction layer (type IV). For simplicity, we only show the recombination processes at the perovskite/electron extraction layer ( $\mathrm{N}$ interface); similar recombination processes occur at the perovskite/hole extraction layer (P interface). Green arrows represent recombination processes, black arrows represent charge-carrier back transfer processes. d| Band-tail recombination losses near the energy band edges. Non-radiative recombination losses are associated with charge carrier relaxation from the energy band edges to the band-tail states via the release of photons. $E_{\mathrm{a}}$ is the activation energy of the trapped electrons. HOMO: highest occupied molecular orbital; LUMO: lowest unoccupied molecular orbital.

Figure 3| Characterization techniques to quantify non-radiative recombination losses in perovskite thin films and complete devices. a| Schematic representation of a photoluminescence quantum yield measurement of a perovskite thin film deposited on a substrate. $\mathbf{b} \mid$ Illustration of the electroluminescence measurement of a complete device. $\mathbf{c |}$ Trap density of states (tDOS) of a complete PSC measured by thermal admittance spectroscopy, $\mathrm{E}_{\omega}$ is the energy demarcation defined by the applied angular frequency $\omega$; band 1,2 and 3 include trap states with energy depths of $0.35-0.40 \mathrm{eV}, 0.40-0.50 \mathrm{eV}$, and 
$>0.50 \mathrm{eV}$, respectively. d| Mapping of quasi-Fermi level splitting (QFLS) for a series of samples consisting of PTAA/Perovskite/ $\mathrm{C}_{60}$, PTAA/perovskite, perovskite/ $\mathrm{C}_{60}$, and perovskite only, revealing the contribution to non-radiative losses of the top and bottom interfaces individually and combined. Each square is $1 \mathrm{~cm}^{2}$. e| Energy histograms for the QFLS of the samples in panel d, showing that the open-circuit voltage deficit induced by each component do not add up in the complete device. All samples were excited with a $450 \mathrm{~nm}$ laser (with intensity equivalent to $1 \mathrm{sun}$ ) and the histograms were recorded on $5 \mathrm{~mm} \times 5 \mathrm{~mm}$ squares in the middle of the samples without photoluminescence quenching, owing to the encapsulation glue. PTAA: poly[bis (4-phenyl)(2,4,6trimethylphenyl)amine]. Part $\mathbf{c}$ adapted with permission from REF. 48. Part $\mathbf{d}$ and $\mathbf{e}$ adapted with permission from REF. 106.

Figure 4| Defect passivation strategies. a| Illustration of a halide perovskite thin film with potassium passivation. The surplus halide is immobilized through complexing with $\mathrm{K}^{+}$into benign compounds at the grain boundaries and at the surface of the perovskite film. $\mathbf{b}$ Chemical structures of some widely used passivation molecules with various functional moieties. $\mathbf{c}$ Impact of hydrogen bonds on passivation. Attenuated total reflection-Fouriertransform infrared spectroscopy (ATR-FTIR) (left) of FAI, EDEA and a FAI:EDEA (1:1) mixture show obvious broadened absorption from $v(\mathrm{~N}-\mathrm{H})$ and $v(\mathrm{C}-\mathrm{H})$ (in the range of 2500-3500 $\mathrm{cm}^{-1}$ ) of the EDEA/FAI mixture compared to those in pure FAI and EDEA. Meanwhile, the $\mathrm{N}-\mathrm{H}$ scissoring vibration $\delta(\mathrm{N}-\mathrm{H})$ absorption (in the range of 1500-1700 $\mathrm{cm}^{-1}$ ) bands greatly weaken in the mixture, suggesting the restriction of $\mathrm{N}-\mathrm{H}$ bending caused by intermolecular interactions. Both observations confirm formation of hydrogen bonds between the passivation molecules and FAI. Calculated electron distribution at the valence band maximum (center) and proton nuclear magnetic resonance (1H NMR) spectra (right) show the difference of hydrogen bonds for various molecules. The chemical shift at $8.9 \mathrm{ppm}$ from pure FAI origins from the resonance from the active protons at its amino groups. However, this peak is broadened and moves to $7.7 \mathrm{ppm}$ and $5.9 \mathrm{ppm}$ by the use of $15 \%$ HMDA and EDEA, respectively, indicating the weaker hydrogen-bonding ability of EDEA with respect to HMDA. d| A summary of the major passivation mechanisms reported to date. FAI, formamidinium iodide; EDEA, 2,2'2(ethylenedioxy)diethylamine. HMDA, hexamethylenediamine, which excludes oxygen atoms. Part a adapted with permission from REF. 13. Part c adapted with permission from REF. 129.

Figure 5| Graded junctions in perovskite solar cells. a| Left, device configuration of a photovoltaic solar cell with a graded junction. Right, the molecular structure of guanidinium bromide and the energy band diagram of the graded junction. $E_{\mathrm{F}}$ is the Fermi level, $\Delta_{1}$ and $\Delta_{2}$ the offsets of the conduction band minimum $\left(E_{\mathrm{c}}\right)$ and valence band maximum $\left(E_{\mathrm{v}}\right)$, respectively. $\Delta E_{\mathrm{g}}$ reflects the difference between and bulk and surface bandgap. $\mathbf{b} \mid$ Transient absorption spectra of films of quartz glass/perovskite/PMMA in the transmission geometry, excited using 400-nm (90-fs) excitation pump pulses from the glass side (top) and from the PMMA side (bottom). The spectra show a wider bandgap close to the top surface region, suppressing non-radiative recombination. $\mathbf{c}$ |Environmental stability of non-encapsulated control and double-layered halide architecture (DHA) devices. The 
inset shows photographs of the devices after 200 hours under $85 \%$ relative humidity at room temperature. d| Operational stability of encapsulated control and DHA devices; the plot is obtained by tracking devices at their maximum power point under 1-sun illumination. ITO, indium tin oxide; PTAA, poly[bis (4-phenyl)(2,4,6-trimethylphenyl)amine]; $\mathrm{PC}_{61} \mathrm{BM}$, [6,6]-phenyl-C61-butyric acid methyl ester; BCP, bathocuproine; PMMA, Poly(methyl methacrylate); $T$, transmission. Part $\mathbf{b}$ adapted with permission from REF. 21. Part $\mathbf{c}$ and $\mathbf{d}$ reproduced with permission from REF. 22.

Figure 6| Calculations of the maximum achievable device performance parameters in single-junction perovskite solar cells. a| Calculated short-circuit current density $\left(J_{\mathrm{sc}}\right)$ as a function of the bandgap of perovskite absorbers and external quantum efficiency (EQE). The white dash line presents an EQE of 95\%, the black line an EQE of 1. Yellow and green dots correspond to photovoltaic solar cells comprising a perovskite with a bandgap of 1.48 $\mathrm{eV}\left(\mathrm{FAPbI}_{3}\right)$ and $1.53 \mathrm{eV}\left(\mathrm{FA}_{0.92} \mathrm{MA}_{0.08} \mathrm{PbI}_{3}\right)$, respectively. b| Calculated open-circuit voltage $\left(V_{\mathrm{oc}}\right)$ as a function of bandgap and external radiative efficiency (ERE). In panel $\mathrm{b}$, $\mathrm{c}$ and $\mathrm{d}$, the white dash line is calculated using a $V_{\text {oc }}$ deficit of $0.31 \mathrm{~V}$, comparable to that of conventional GaAs solar cells. c| Calculated fill factor as a function of bandgap and ERE. d|Calculated power conversion efficiency (PCE) as a function of bandgap and ERE.

\section{Box 1| Introduction to metal halide perovskites}

Perovskite compounds are a large family of crystalline materials with the structure of the mineral calcium titanate. They are named after Russian mineralogist Lev Perovski. Typical metal halide perovskites have a $3 \mathrm{D}$ crystal structure with general formula $\mathrm{ABX}_{3}$, where $\mathrm{A}$ is a monovalent cation ( $\operatorname{such}$ as $\mathrm{FA}^{+}=$formamidinium, $\mathrm{MA}^{+}=$methylammonium or $\mathrm{Cs}^{+}$), $\mathrm{B}$ a divalent metal cation ( $\mathrm{such}$ as $\mathrm{Pb}^{2+}$ or $\mathrm{Sn}^{2+}$ ), and $\mathrm{X}$ a halogen anion (such as $\mathrm{I}^{-}, \mathrm{Br}^{-}$or $\mathrm{Cl}^{-}$), as illustrated in the figure. Their crystallographic stability and probable structure are governed by the Goldschmidt tolerance factor $(t)$ and octahedral factor $(v)$ : $t=$ $\left(R_{\mathrm{A}}+R_{\mathrm{X}}\right) / \sqrt{ } 2\left(R_{\mathrm{B}}+R_{\mathrm{X}}\right)$, where $R_{\mathrm{A}}, R_{\mathrm{B}}$ and $R_{\mathrm{X}}$ are the ionic radii of the corresponding ions; $v$ $=R_{\mathrm{B}} / R_{\mathrm{X}}$. To preserve the $3 \mathrm{D}$ structure of perovskites, $t$ and $v$ typically have to be in the range of $0.81<t<1.11$ and $0.44<v<0.90$, respectively. Otherwise, the material is in the Ruddlesden-Popper phase $\left(\mathrm{R}_{2} \mathrm{~A}_{\mathrm{n}-1} \mathrm{~B}_{\mathrm{n}} \mathrm{X}_{3 \mathrm{n}+1}\right)$, that is, has a $2 \mathrm{D}$ or quasi-2D perovskite 
structure, in which the size of the organic bulky cation $(\mathrm{R})$ is beyond the limit of the Goldschmidt tolerance factor ( $\mathrm{n}$ is the number of octahedra layers).

In general, perovskites with different dimensionalities have strikingly different physical and chemical properties. Notably, metal halide perovskites can be synthesized as highquality polycrystalline thin films using scalable solution fabrication processes, and exhibit high optical absorption coefficients, long carrier diffusion lengths and a high tolerance for intrinsic defects. In addition, their bandgaps are easily tunable by varying the chemical composition of the precursor solutions. 